\title{
Frequency of Subclinical Hypothyroidism in Old-age Population
}

\author{
Khalil Ullah Shabir, Kashif Ullah Shabir, Maria Qadri, Uzair Yaqoob, Syed Muhammad Ahsan Nawaz and \\ Balvender Singh \\ Department of Medicine, Jinnah Postgraduate Medical Centre, Karachi, Pakistan
}

\begin{abstract}
This study was conducted to determine the frequency of subclinical hypothyroidism ( $\mathrm{SCH}$ ) in the old age population (>60 years). A cross-sectional study was conducted from July 2019 to January 2020 at Jinnah Postgraduate Medical Centre, Karachi, Pakistan. Healthy subjects (having no active complaint) of either sex aged 60-80 years were included. Those already on thyroid replacement, preexisting thyroid diseases, prior radiation therapy, or having chronic kidney disease were excluded. A total of 133 participants, 79 males and 54 females, were selected with a mean age of $69.5 \pm 6.4$ years. There were 29 diabetics, 30 hypertensives, and 10 smokers. A total of $17(12.8 \%)$ were found to have $\mathrm{SCH}$. On stratification, gender, diabetes mellitus, and hypertension had a significant association with the presence of $\mathrm{SCH}$. SCH is a modifiable risk factor, more in females, diabetics, hypertensives, and the old age population. These results highlight the need for screening for $\mathrm{SCH}$ in primary care settings, especially in old age.
\end{abstract}

Key Words: Subclinical hypothyroidism, Old age, Gender, Diabetes, Hypertension.

How to cite this article: Shabir KU, Shabir KU, Qadri M, Yaqoob U, Nawaz SMA, Singh B. Frequency of Subclinical Hypothyroidism in Old-age Population. J Coll Physicians Surg Pak 2021; 31(08):995-997.

Subclinical hypothyroidism $(\mathrm{SCH})$ is a disorder of the thyroid gland, characterised by elevated thyroid-stimulating hormone (TSH), with normal levels of free $\mathrm{T}_{3}\left(\mathrm{FT}_{3}\right)$ and free $\mathrm{T}_{4}\left(\mathrm{FT}_{4}\right)$. Due to the varying clinical presentation, the only way to diagnose it is through biochemical testing. ${ }^{1}$ Etiology is usually similar to that of simple hypothyroidism, most common being Hashimoto's thyroiditis; whereas, other causes include subacute thyroiditis, postpartum thyroiditis, previous hyperthyroidism, autoimmune diseases, thyroid injury/inflammation (due to radiation, surgery, or medication), and thyroid infiltration. Before making a definitive diagnosis of $\mathrm{SCH}$, other causes of a raised TSH must be excluded, like recovery from non-thyroidal illness, thyroid hormone resistance, radiation, prior thyroid diseases like a cold or hot nodule, or chronic kidney disease. ${ }^{1}$

Signs and symptoms of SCH are non-specific and non-diagnostic. However, in old age, patients may present with clinical features of depression and cognitive dysfunction, fatigue, constipation, dyslipidemia (elevated low-density lipoprotein), and cardiac dysfunction (bradycardia). ${ }^{1}$ It is also associated with an increased risk of coronary heart disease (CHD). ${ }^{2}$

Correspondence to: Dr. Uzair Yaqoob, Department of Medicine, Jinnah Postgraduate Medical Centre, Karachi, Pakistan

E-mail: ozair_91393@hotmail.com

Received: February 10, 2021; Revised: May 04, 2021;

Accepted: May 24, 2021

DOI: https://doi.org/10.29271/jcpsp.2021.08.995
The decision to treat the patients with $\mathrm{SCH}$ remains controversial, with some physicians in favour, irrespective of symptoms, while the majority agreeing to treat only if they are symptomatic, TSH level is $>10 \mathrm{mlU} / \mathrm{L}$, anti-TPO antibodies positive, with or without any evidence of CHD. ${ }^{3}$

The local data related to $\mathrm{SCH}$ is insufficient. The objective of this study is to determine the prevalence of $\mathrm{SCH}$ in patients $>60$ years and evaluate its relationship with factors like age, gender, smoking status, and comorbidities (diabetes and hypertension). This will highlight the importance of screening for thyroid diseases, especially in the elderly, to start early management.

This is an observational, cross-sectional study, carried out at the Medicine Department, Jinnah Postgraduate Medical Centre, Karachi, Pakistan. The study duration was from July 15, 2019 to January 15, 2020. After receiving the patients in the Outpatient Department, informed consents were taken.

A non-probability consecutive sampling technique was used for data collection in healthy subjects (having no active complaint) of either sex aged 60-80 years. Their reason for consultation was not a thyroid-related illness, but a regular health checkup. Patients already on thyroid replacement, preexisting thyroid diseases, prior radiation therapy, or those having chronic kidney disease were excluded.

The thyroid function assessment was done by measurement of FT3, FT4, and TSH, using a chemiluminescence immunoassay (CLIA) kit. SCH was defined as a condition in which the TSH level is greater than $4.5 \mathrm{mlU} / \mathrm{L}$ in the presence of a normal level of 
peripheral free $\mathrm{T} 3$ and $\mathrm{T} 4$. The patients were treated as per hospital protocol. All the data were collected through a predesigned proforma.

The statistical analysis was performed using SPSS version 22 (IBM Corp., Armonk, NY, US). Mean and standard deviations were calculated for age; while frequency and percentages were calculated for gender, smoking, diabetes mellitus (DM), hypertension (HTN), and subclinical hypothyroidism. Effect modifiers and confounders were controlled by stratification, according to age, gender, smoking, DM, and HTN. Post-stratification, the ChiSquare test or Fisher's Exact test was applied. A p-value $\leq 0.05$ was considered significant.

A total of 133 healthy subjects were selected for this study, having a mean age of $69.5 \pm 6.4$ years. Among cases, 79 (59.4\%) were males and $54(40.6 \%)$ were females. When the age groups were made into classes, $70(52.6 \%)$ were in the age group of $60-70$ years, while $63(47.4 \%)$ were in that of $71-80$ years.

There were 29 (21.8\%) diabetics, 30 (22.6\%) hypertensives, and $10(7.5 \%)$ smokers. A total of $17(12.8 \%)$ patients were found to have $\mathrm{SCH}$.

By stratification of $\mathrm{SCH}$, it was found that gender, DM, and HTN had a significant association with the presence of subclinical hypothyroidism $(p=0.030, p<0.001$, and $p=0.001$ respectively, Table I).

Table I: Stratification of subclinical hypothyroidism, according to gender, age, smoking, and comorbidities (133).

\begin{tabular}{|c|c|c|c|c|}
\hline & \multicolumn{2}{|c|}{ Subclinical hypothyroidism } & \multirow{2}{*}{ Total } & \multirow{2}{*}{ p-value } \\
\hline & Yes $(n=17)$ & No $(n=116)$ & & \\
\hline \multicolumn{4}{|l|}{ Age (years): } & \multirow{3}{*}{0.125} \\
\hline $60-70$ years & $6(8.6 \%)$ & 64 (91.4\%) & 70 & \\
\hline $71-80$ years & $11(17.5 \%)$ & $52(82.5 \%)$ & 63 & \\
\hline \multicolumn{4}{|l|}{ Gender: } & \multirow{3}{*}{0.030} \\
\hline Male & $6(7.6 \%)$ & $73(92.4 \%)$ & 79 & \\
\hline Female & $11(20.4 \%)$ & $43(79.6 \%)$ & 54 & \\
\hline \multicolumn{4}{|l|}{ Diabetes: } & \multirow{3}{*}{$<0.001$} \\
\hline Yes & $11(37.9 \%)$ & $18(62.1 \%)$ & 29 & \\
\hline No & $6(5.8 \%)$ & $98(94.2 \%)$ & 104 & \\
\hline \multicolumn{4}{|c|}{ Hypertension: } & \multirow{3}{*}{0.001} \\
\hline Yes & $10(33.3 \%)$ & $20(66.7 \%)$ & 30 & \\
\hline No & $7(6.8 \%)$ & $96(93.2 \%)$ & 103 & \\
\hline \multicolumn{4}{|l|}{ Smoking: } & \multirow{3}{*}{0.118} \\
\hline Yes & $3(30.0 \%)$ & $7(70.0 \%)$ & 10 & \\
\hline No & $14(11.4 \%)$ & $109(88.6 \%)$ & 123 & \\
\hline
\end{tabular}

The frequency of $\mathrm{SCH}$ in our hospital-based study population was found to be $12.8 \%$.

On gender-based analysis, the proportion of females affected by $\mathrm{SCH}$ was higher ( $20.4 \%$ vs $7.6 \%)$. This is similar to every major study done on hypothyroidism prevalence. ${ }^{4}$ However, this is very low compared to other studies like Surks et al., where it was concluded that with increased age, there is an increased level of TSH found in the normal population with $\mathrm{SCH}$, approaching $14.5 \% .{ }^{5}$ This shows how variable the prevalence rates of $\mathrm{SCH}$ are in different countries. Evidence shows that in European countries, $\mathrm{SCH}$ is more prevalent in iodine sufficient areas. Moreover, in a study done in Colorado, subjects taking thyroid medication had a $17.6 \%$ prevalence of $\mathrm{SCH}$, compared to those without the medication (9\%), ${ }^{4,6}$ On the other hand, iodine deficiency has been a public health problem in the Eastern Mediterranean countries, like Afghanistan, Algeria, Iraq, Iran, Morocco, Saudi Arabia, Sudan, Yemen, and Pakistan. ${ }^{4}$ This may bethereason behind the low rates of $\mathrm{SCH}$ in these countries. Our results may have overestimated the actual prevalence since they are from older adults.

Diabetics are more likely to have $\mathrm{SCH}$, when compared with the normal population with a higher ratio of diabetic complications. Similarly, hypertensives are more likely to have $\mathrm{SCH} .{ }^{4}$ When $\mathrm{SCH}$ status, according to the comorbid, were assessed in our study, HTN and DM were found to be significantly associated with SCH as well $(p=0.001$, and $p<0.001)$.

These results are limited by the fact being from a single healthcare setup and not the general population; and the same sampling error is the reason for having a high number of hypertensives and diabetics. Moreover, a regular follow-up is required to rule out any TSH measuring lab error or determining the actual cause of raisedTSH.

Studies with a larger sample size assessing people of all ages and populations of both urban and rural settings are needed to have a generalised estimated prevalence in all age groups.

Subclinical hypothyroidism is a modifiable risk factor, more in females, diabetics, hypertensives, and the old age population. These results highlight the need for screening for $\mathrm{SCH}$ in primary care settings, especially in old age adults.

\section{ETHICALAPPROVAL:}

The study was approved by the Institutional Review Board of Jinnah Postgraduate Medical Centre, Karachi, Pakistan.

\section{PATIENTS' CONSENT:}

Informed consents were obtained from the patients to publish the data concerning this case.

\section{CONFLICT OF INTEREST:}

The authors declared no conflict of interest.

\section{AUTHORS' CONTRIBUTION:}

KUS, KUS, MQ: Conception and design, interpretation, drafting, critical revision, final approval.

UY: Data acquisition and analysis, interpretation, drafting, critical revision, final approval.

SMAN: Interpretation, drafting, critical revision, final approval. BS: Analysis and interpretation, drafting, critical revision, final approval.

\section{REFERENCES}

1. Patil N, Rehman A, Jialal I. Hypothyroidism. In: StatPearls [Internet]. Treasure Island (FL): StatPearls Publishing; 2020. Available from: http://www.ncbi.nlm.nih.gov/books/ NBK519536.

2. Rodondi N, den Elzen WPJ, Bauer DC, Cappola AR, Razvi S, Walsh JP, et al. Subclinical hypothyroidism and the risk of coronary heart disease and mortality. JAMA 2010; 
304(12):1365-74. doi: 10.1001/jama.2010.1361

3. Andersen MN, Olsen AMS, Madsen JC, Faber J, TorpPedersen C, Gislason GH, et al. Levothyroxine Substitution in Patients with Subclinical Hypothyroidism and the Risk of Myocardial Infarction and Mortality. PLoS One 2015; 10(6):1-17. doi: 10.1371/journal.pone.0129793

4. Al Eidan E, Rahman S, Al Qahtani S, Al Farhan Al, Abdulmajeed I. Prevalence of subclinical hypothyroidism in adults visiting primary health-care setting in Riyadh. J community Hosp Intern Med Perspect 2018; 8(1):11-5. doi: 10.1080/20009666.2017.1422672.

5. Surks MI, Hollowell JG. Age-specific distribution of serum thyrotropin and antithyroid antibodies in the US population: implications for the prevalence of subclinical hypothyroidism. J Clin Endocrinol Metab 2007; 92(12): 4575-82. doi: 10.1080/20009666.2017.1422672.

6. Canaris GJ, Manowitz NR, Mayor G, Ridgway EC. The Colorado thyroid disease prevalence study. Arch Intern Med 2000; 160(4):526-34. doi: 10.1001/archinte.160.4.526. 